\title{
Application of Magnetic Particle Imaging to Pulmonary Imaging Using Nebulized Magnetic Nanoparticles
}

\author{
Kohei Nishimoto, Atsushi Mimura, Marina Aoki, Natsuo Banura, Kenya Murase \\ Department of Medical Physics and Engineering, Division of Medical Technology and Science, Faculty of Health \\ Science, Graduate School of Medicine, Osaka University, Osaka, Japan \\ Email: murase@sahs.med.osaka-u.ac.jp
}

Received 17 April 2015; accepted 22 May 2015; published 25 May 2015

Copyright (C) 2015 by authors and Scientific Research Publishing Inc.

This work is licensed under the Creative Commons Attribution International License (CC BY). http://creativecommons.org/licenses/by/4.0/

(c) (i) Open Access

\begin{abstract}
Purpose: To investigate the feasibility of applying magnetic particle imaging (MPI) to pulmonary imaging using nebulized magnetic nanoparticles (MNPs) and to quantify the mucociliary clearance in the lung, using small animal experiments. Materials and Methods: Intrapulmonary administration of MNPs was performed in seven-week-old male ICR (Institute of Cancer Research) mice $(n=8)$ using a nebulized microsprayer connected to a high-pressure syringe containing $50 \mu \mathrm{L}$ of MNPs (500 mM Resovist $\circledast$ ). We imaged the lungs using our MPI scanner 2.5 hours, 1 day, 3 days, and 7 days after the intrapulmonary administration of MNPs. The average MPI value was calculated by drawing a region of interest (ROI) on the lungs by taking the threshold value for extracting the contour as $20 \%$ of the maximum MPI value within the ROI. The MPI value was defined as the pixel value of the transverse image reconstructed from the third-harmonic signals. Mice were sacrificed immediately after the last MPI and X-ray CT studies on day 7, and 5 lobes of the lung in each mouse were extracted to confirm the accumulation of iron using Berlin blue staining. Results: We could visualize the distribution of MNPs in the lungs as positive contrast using MPI with use of nebulized MNPs. The presence of iron in the lung was confirmed by Berlin blue staining. The average MPI value decreased with time and tended to saturate. The clearance rate was calculated to be 0.505 $d^{-1}$ from the time course of the average MPI value in the lungs. Conclusion: Our preliminary results suggest that MPI can be applied to pulmonary imaging by nebulizing MNPs and can be useful for quantifying the mucociliary clearance in the lung.
\end{abstract}

\section{Keywords}

Magnetic Particle Imaging, Pulmonary Imaging, Nebulized Magnetic Nanoparticles, Animal Experiments 


\section{Introduction}

Recently, a magnetic targeting method has been proposed for localizing drug carriers such as liposomes containing both magnetic nanoparticles (MNPs) and drugs in the objective organ or tissue by applying an external magnetic field, and it attracts attention as a next-generation therapeutic strategy for cancer [1] [2]. The application of this approach with use of nebulized MNPs to lung diseases has also been considered [1]. The development of a method to specifically image MNPs is desired to realize this therapeutic strategy.

Targeted pulmonary inhalation aerosol-based delivery facilitates the direct application of drugs to the lungs in a controlled manner, and has inherent advantages such as a fast onset of pharmaceutical action, higher local concentration of drugs, and reduced systemic side effects [3]. However, rapid mucociliary clearance of trapped drug carriers will put a serious limitation to any attempt of designing inhalable controlled release systems [4]. Thus, the development of a method to simply and quantitatively monitor the mucociliary clearance of drug carriers is also desired to enhance the usefulness of the aerosol-based approach of drug delivery.

Recently, a new imaging method called magnetic particle imaging (MPI) has been introduced [5]. MPI allows imaging of the spatial distribution of MNPs with high sensitivity, spatial resolution, and imaging speed [5]. MPI uses the nonlinear response of MNPs to detect their presence in an alternating magnetic field, which is referred to here as the drive magnetic field. Spatial encoding is accomplished by saturating the MNPs over most of the imaged region using a static magnetic field (selection magnetic field), except in the vicinity of a special position called the field-free point [5] or field-free line [6]. We have developed a system for MPI with a field-free-line encoding scheme, in which the field-free line is generated using two opposing neodymium magnets, and transverse images are reconstructed from the third-harmonic signals received by a gradiometer coil using the maximum likelihood-expectation maximization (ML-EM) algorithm [7] [8].

The purpose of this study was to investigate the feasibility of applying MPI to pulmonary imaging using nebulized MNPs and to quantify the mucociliary clearance in the lung, using small animal experiments.

\section{Materials and Methods}

\subsection{Magnetic Nanoparticles}

In this study, Resovist ${ }^{\circledR}$ was used as the source of MNPs and was purchased from Fuji Film RI Pharma Co. (Tokyo, Japan). Resovist ${ }^{\circledR}$ consists of iron oxide (maghemite, $\gamma-\mathrm{Fe}_{2} \mathrm{O}_{3}$ ) coated with carboxydextran [9]-[11]. It is an organ-specific contrast agent for magnetic resonance imaging, used especially for the detection and characterization of small focal liver lesions.

\subsection{Magnetic Particle Imaging}

Figure 1 shows a photograph of our MPI scanner. The details of our MPI system are described in our previous

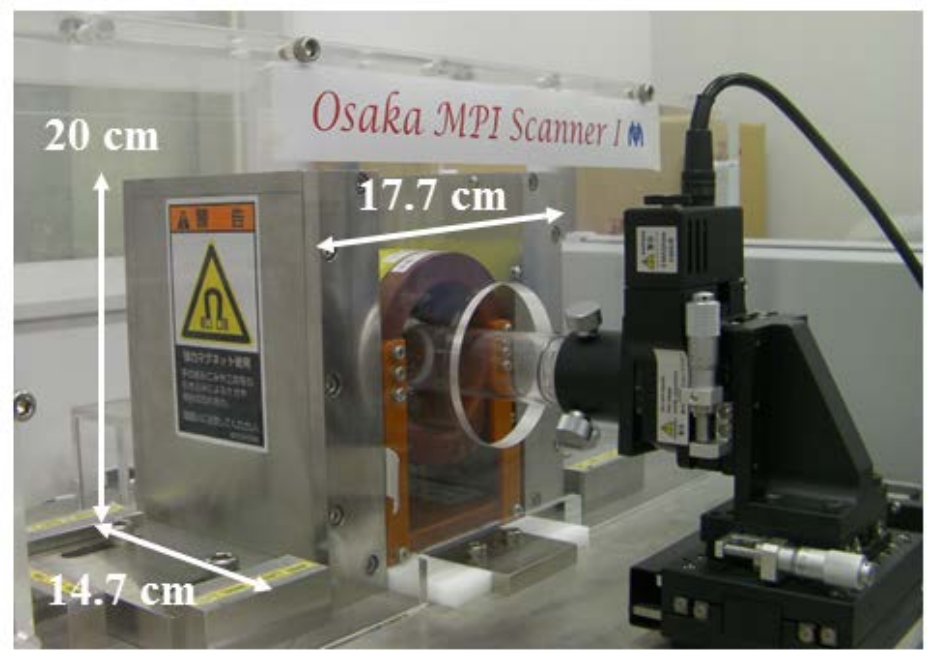

Figure 1. Photograph of our scanner for magnetic particle imaging (MPI). 
paper [7] [8]. In brief, a drive magnetic field was generated using an excitation coil (solenoid coil $100 \mathrm{~mm}$ in length, $80 \mathrm{~mm}$ in inner diameter, and $110 \mathrm{~mm}$ in outer diameter). AC power was supplied to the excitation coil by a programmable power supply (EC1000S, NF Co., Yokohama, Japan), and was controlled using a sinusoidal wave generated by a digital function generator (DF1906, NF Co., Yokohama, Japan). The frequency and peakto-peak strength of the drive magnetic field were taken as $400 \mathrm{~Hz}$ and $20 \mathrm{mT}$, respectively. The signal generated by MNPs was received by a gradiometer coil $(50 \mathrm{~mm}$ in length, $35 \mathrm{~mm}$ in inner diameter, and $40 \mathrm{~mm}$ in outer diameter), and the third-harmonic signal was extracted using a preamplifier (T-AMP03HC, Turtle Industry Co., Ibaragi, Japan) and a lock-in amplifier (LI5640, NF Co., Yokohama, Japan). The output of the lock-in amplifier was converted to digital data by a personal computer connected to a multifunction data acquisition device with a universal serial bus port (USB-6212, National Instruments Co., TX, USA). The sampling time was taken as 10 ms. When measuring signals using the gradiometer coil, a sample was placed $12.5 \mathrm{~mm}$ (i.e., one quarter of the coil length) from the center of the gradiometer coil and the coil, including the sample, was moved such that the center of the sample coincided with the position of the field-free line. The selection magnetic field was generated by two opposing neodymium magnets (Neomax Engineering Co., Gunma, Japan). The field-free line can be generated at the center of the two neodymium magnets.

To acquire projection data for image reconstruction, a sample (mouse) in the receiving coil was automatically rotated around the z-axis over $180^{\circ}$ in steps of $5^{\circ}$ and translated in the x-direction from $-16 \mathrm{~mm}$ to $16 \mathrm{~mm}$ in steps of $1 \mathrm{~mm}$, using an XYZ-axes rotary stage (HPS80-50X-M5, Sigma Koki Co., Tokyo, Japan), which was controlled using LabVIEW (National Instruments Co., TX, USA). Data acquisition took about 12 min. Each projection data set was then transformed into 64 bins by linear interpolation. Both the inhomogeneous sensitivity of the receiving coil and feed through interference were corrected using the method described in [12]. Transverse images were reconstructed from the projection data using the ML-EM algorithm over 15 iterations, in which the initial concentration of MNPs was assumed to be uniform [7] [8].

\subsection{Animal Experiments}

Seven-week-old male ICR (Institute of Cancer Research) mice $(\mathrm{n}=8)$ weighing $35.5 \pm 2.4 \mathrm{~g}($ mean \pm standard deviation) were purchased from Charles River Laboratories Japan, Inc. (Yokohama, Japan), and were habituated to the rearing environment for one week before the experiment. The animals had free access to food and water, and were kept under standard laboratory conditions of $22^{\circ} \mathrm{C}-23^{\circ} \mathrm{C}$ room temperatures, around $50 \%$ humidity, and a 12:12 hour light/dark cycle.

After habituation, intrapulmonary administration of MNPs was performed using a nebulizing microsprayer (Penn-Century Inc., PA, USA) connected to a high-pressure syringe containing $50 \mu \mathrm{L}$ of MNPs (500 mM Resovist $^{\circledR}$ ) under anesthesia by pentobarbital sodium (Somnopentyl, Kyoritsu Seiyaku Co., Tokyo, Japan) (0.012 $\mathrm{mL} / \mathrm{g}$ body weight). At the intrapulmonary administration of MNPs, the tip of the microsprayer was introduced into the trachea of a mouse using a dedicated laryngoscope. We imaged the lungs using our MPI scanner [7] [8] 2.5 hours, 1 day, 3 days, and 7 days after the intrapulmonary administration of MNPs. In this study, one slice of the MPI image with the maximum signal intensity was obtained per mouse. After the MPI studies, X-ray CT images were obtained using a 4-row multi-slice CT scanner (Asteion, Toshiba Medical Systems Co., Tochigi, Japan) with a tube voltage of $120 \mathrm{kV}$ and a tube current of $210 \mathrm{~mA}$. The MPI image was co-registered with the $\mathrm{X}$-ray CT image using parameters for magnification and rotation that were previously obtained using a phantom with 3 point sources with a diameter of $0.5 \mathrm{~mm}$ and filled with $100 \mathrm{mM}$ MNPs. Mice were sacrificed immediately after the last MPI and X-ray CT studies on day 7, and 5 lobes of the lung in each mouse were extracted to confirm the accumulation of iron using Berlin blue staining.

All animal experiments described above were approved by the animal ethics committee at Osaka University School of Medicine.

\subsection{Data Analysis}

In this study, the MPI value was defined as the pixel value of the transverse image reconstructed from the third-harmonic signals. We calculated the average MPI value within a region of interest (ROI) drawn on the lung by taking the threshold value for extracting the contour as $20 \%$ of the maximum MPI value in the ROI.

The normalized average MPI value was calculated by dividing the average MPI value by that on day 0 , and was expressed as the mean \pm standard error for $n=8$. We calculated the clearance rate $(k)$ and the normalized 
average MPI value at infinite time $\left(M_{\text {inf }}\right)$ by fitting the time course of the normalized average MPI value $(M(t))$ to the following equation:

$$
M(t)=\left(1-M_{\mathrm{inf}}\right) e^{-k t}+M_{\mathrm{inf}}
$$

In this study, we used the Simplex method [13] for curve fitting.

\section{Results}

Figure 2 shows the typical fusion images between the MPI and X-ray CT images obtained at 2.5 hours, 1 day, 3 days, and 7 days after the intrapulmonary administration of MNPs. Note that the lowest and highest levels for display were set at the same in all the MPI images. As shown in Figure 2, the pixel values of the MPI images decreased with time. It should be noted that there was an excellent linear correlation between the pixel value of the MPI image and the concentration of Resovist ${ }^{\mathbb{B}}$ with a correlation coefficient of 0.998 (plot not shown).

Figure 3 shows the typical photomicrographs of Berlin blue staining in 5 lobes of the lung extracted on day 7 . As shown by blue dots in Figure 3, MNPs were observed in all lobes of the lung.

Figure 4 shows the time course of the normalized average MPI value in the lung. As shown in Figure 4, the normalized average MPI value decreased with time and tended to saturate. This time course was well described by Equation (1) $(\mathrm{r}=0.995)$, and $k$ and $M_{\text {inf }}$ in Equation (1) were calculated to be 0.505 day $^{-1}$ and 0.506 , respectively.

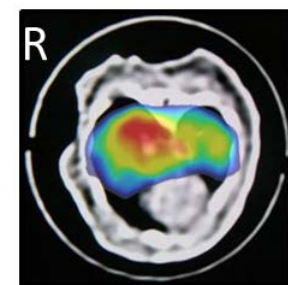

2.5 hours

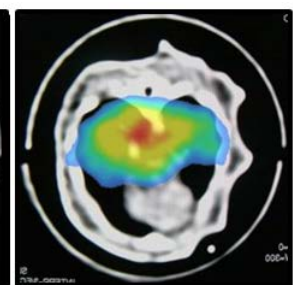

1 day

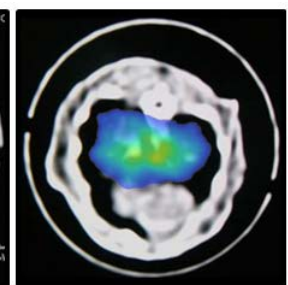

3 days

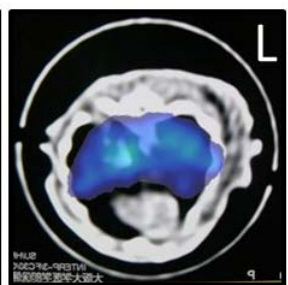

7 days

Figure 2. Typical example of the MPI images of the lung obtained at 2.5 hours, 1 day, 3 days, and 7 days after the intrapulmonary administration of magnetic nanoparticles (MNPs) $\left(50 \mu \mathrm{L}\right.$ of $500 \mathrm{mM}$ Resovist $\left.{ }^{\mathbb{R}}\right)$ using a nebulized microsprayer connected with a high-pressure syringe under anesthesia, which were superimposed on the X-ray CT images. Note that the lowest and highest levels for display were set at the same in all the MPI images.

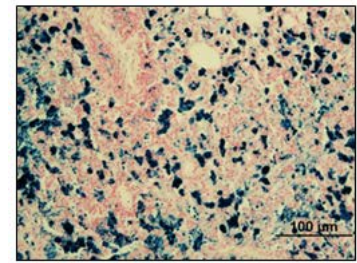

L

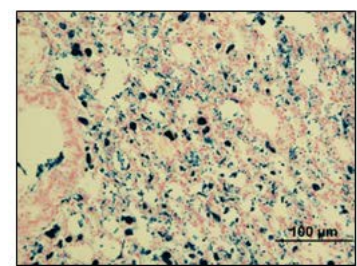

RP

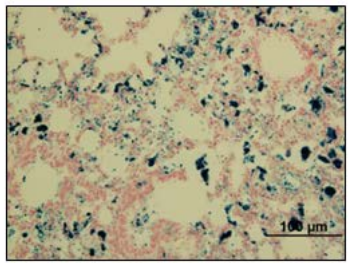

RA

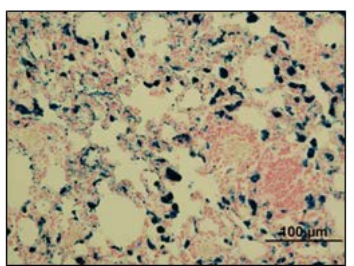

RAC

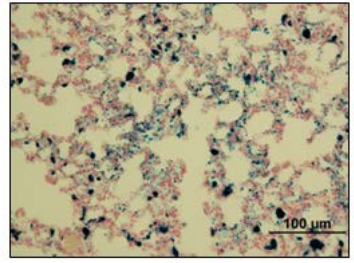

RM

L: Left lobe

RA: Right anterior lobe

RM: Right middle lobe

RP: Right posterior lobe

RAC: Right accessory lobe

Figure 3. Typical example of the photomicrographs of Berlin blue staining in the left (L), right anterior (RA), right middle (RM), right posterior (RP), and right accessory (RAC) lobes of the lung. Magnification 200×; scale bar $=100 \mu \mathrm{m}$. 


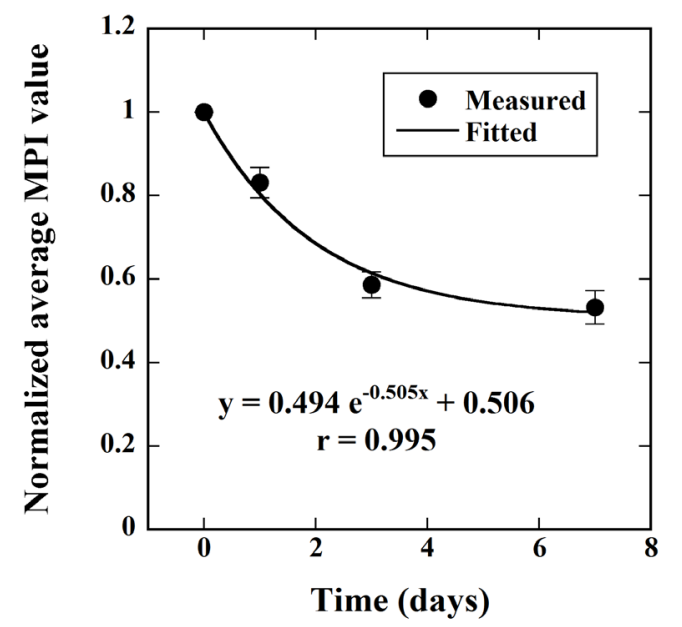

Figure 4. Time course of the average MPI value, from which the clearance rate $(k)$ and the normalized average MPI value at infinite time $\left(M_{\text {inf }}\right)$ of the MNPs in the lung was calculated using Equation (1). The MPI value was defined as the pixel value of the transverse image reconstructed from the third-harmonic signals, and the average MPI value was calculated by drawing a region of interest (ROI) on the lung by taking the threshold value for extracting the contour as $20 \%$ of the maximum MPI value in the ROI. Data are represented by mean \pm standard error for $n=8$. Note that the average MPI value was normalized by that on day 0 .

\section{Discussion}

In this study, we investigated the feasibility of applying MPI to pulmonary imaging in mice using nebulized MNPs. Our preliminary results (Figure 2) suggest that MPI can be applied to pulmonary imaging using nebulized MNPs. To the best of our knowledge, this is the first report on the application of MPI to pulmonary imaging.

As shown in Figure 2, we could successfully visualize the regional distribution of MNPs in the lung as positive contrast using MPI with use of nebulized MNPs. This excellent visibility of MPI appears to be useful for in vivo evaluation of magnetic drug targeting and/or aerosol-based drug delivery [1] [2].

The time course of the average MPI value (Figure 4) was very similar to the retention curve of iridium (Ir)192 nanoparticles with 17 - to 20 -nm median diameter in the total lung of rats measured using single photon emission computed tomography [14]. Semmler et al. [15] reported that Ir-192 nanoparticles were cleared predominantly by mucociliary clearance from the peripheral lung via the airways and larynx into the gastrointestinal tract and were found in feces, and that the fecal excretion observed throughout the entire study of retention during 6 months confirmed the predominance of this clearance pathway. Although we did not confirm the presence of MNPs in feces in this study, we speculate that the time-dependent decrease in the pixel value of the MPI image (Figure 2 and Figure 4) is mainly due to the mucociliary clearance via the same pathway as that reported by Semmler et al. [15].

A limitation of this study is that the MPI value was obtained from a single slice of the MPI image with the maximum signal intensity. For more detailed analysis, three-dimensional (multi-slice) data will be necessary. If three-dimensional data were obtained, the regional mucociliary clearance in the lung could be evaluated in a three-dimensional manner. These studies are currently in progress.

Techniques using radioisotopes such as technetium-99m are widely used to measure mucociliary clearance [16]. The observation time in these studies, however, is limited by the short half-life of the radioisotopes. In limited cases, clearance of inhaled nanoparticles from the rat lung has been measured over 6 months using radioisotopes with longer half-life such as Ir-192 [14] [15] as mentioned above. Similar studies may be possible using magnetic resonance imaging (MRI) after inhalation of superparamagnetic iron oxide. When we attempted to image MNPs using MRI with a conventional transverse relaxation time $\left(T_{2}^{*}\right)$-weighted imaging sequence, it was almost impossible due to large susceptibility-induced MR signal loss and image distortions in the regions near the MNPs. Recently, however, MRI pulse sequences capable of preserving the signal from spins with ul- 
trashort $T_{2}{ }^{*}$, such as ultrashort echo time (UTE) [17] and sweep imaging with Fourier transformation (SWIFT) sequences [18], have been developed. With these pulse sequences, MNPs can be detected and quantified based on the shortening of the longitudinal relaxation time of water $\left(T_{1}\right)$ [18]. Zhang et al. [19] reported that the reciprocal of $T_{1}$ measured using the SWIFT sequence combined with the Look-Locker method has a linear relationship with MNPs concentration up to $53.6 \mathrm{mM}$ of iron. Although their method also appears to be promising and useful for quantifying the concentration of MNPs in the lung, significant research and technology development remain to be done before establishing the effectiveness of their method when translated to animal and/or human studies [20].

As previously described, Resovist ${ }^{\circledR}$ has been used for years in the clinical setting as a contrast agent for MRI. Thus, its biocompatibility is well documented. Furthermore, the compatibility of MNPs with lung deposition via inhalation has been demonstrated in several studies [21]. In addition, some occupational groups, such as welders, who are exposed to relatively high doses of iron oxide dust, apparently do not suffer from the toxicity related to iron oxide [22]. In this study, we injected $50 \mu \mathrm{L}$ of Resovist ${ }^{\mathbb{R}}$ at a concentration of $500 \mathrm{mM}$ into the trachea of a mouse using a nebulizing microsprayer. We selected this dose in consideration of the signal-to-noise ratio of the MPI image. Although we did not observe the apparent side effects induced by this injection in mice during experiments, an effort to reduce the dose will be necessary for enhancing the safety of this method.

\section{Conclusion}

Our preliminary results suggest that MPI can be applied to pulmonary imaging using nebulized MNPs and will provide a new imaging strategy not only for quantifying the mucociliary function in the lung but also for establishing reliable and safe magnetic drug targeting and/or aerosol-based drug delivery in the clinical setting.

\section{Acknowledgements}

This work was supported by a Grant-in-Aid for Scientific Research (B) (Grant No. 25282131) from the Japan Society for the Promotion of Science (JSPS).

\section{Declaration of Interest}

The authors report no conflicts of interest. The authors alone are responsible for the content and writing of the paper.

\section{References}

[1] Dames, P., Gleich, B., Flemmer, A., Hajek, K., Seidl, N., Wiekhorst, F., Eberbeck, D., Bittmann, I., Bergemann, C., Weyh, T., Trahms, L., Rosenecker, J. and Rudolph, C. (2007) Targeted Delivery of Magnetic Aerosol Droplets to the Lung. Nature Nanotechnology, 2, 495-499. http://dx.doi.org/10.1038/nnano.2007.217

[2] Mikhaylov, G., Mikac, U., Magaeva, A.A., Itin, V.I., Naiden, E.P., Psakhye, I., Babes, L., Reinheckel, T., Peters, C., Zeiser, R., Bogyo, M., Turk, V., Psakhye, S.G., Turk, B. and Vasilieva, O. (2011) Ferri-Liposomesas an MRI-Visible Drug-Delivery System for Targeting Tumours and Their Microenvironment. Nature Nanotechnology, 6, $594-602$. http://dx.doi.org/10.1038/nnano.2011.112

[3] Stocke, N.A., Meenach, S.A., Arnold, S.M., Mansour, H.M. and Hilt, J.Z. (2015) Formulation and Characterization of Inhalable Magnetic Nanocomposite Microparticles (MnMs) for Targeted Pulmonary Delivery via Spray Drying. International Journal of Pharmaceutics, 479, 320-328. http://dx.doi.org/10.1016/j.ijpharm.2014.12.050

[4] Kirch, J., Guenther, M., Doshi, N., Schaefer, U.F., Schneider, M., Mitragotri, S. and Lehr, C-M. (2012) Mucociliary Clearance of Micro- and Nanoparticles Is Independent of Size, Shape and Charge-An ex Vivo and in Silico Approach. Journal of Controlled Release, 159, 128-134. http://dx.doi.org/10.1016/j.jconrel.2011.12.015

[5] Gleich, B. and Weizenecker, J. (2005) Tomographic Imaging Using the Nonlinear Response of Magnetic Particles. Nature, 435, 1214-1217. http://dx.doi.org/10.1038/nature03808

[6] Goodwill, P.W., Konkle, J.J., Zheng, B., Saritas, E.U. and Conolly, S.M. (2012) Projection X-space Magnetic Particle Imaging. IEEE Transactions on Medical Imaging, 31, 1076-1085. http://dx.doi.org/10.1109/TMI.2012.2185247

[7] Murase, K., Hiratsuka, S., Song, R. and Takeuchi, Y. (2014) Development of a System for Magnetic Particle Imaging Using Neodymium Magnets and Gradiometer. Japanese Journal of Applied Physics, 53, Article ID: 067001. http://dx.doi.org/10.7567/JJAP.53.067001

[8] Murase, K., Song, R. and Hiratsuka, S. (2014) Magnetic Particle Imaging of Blood Coagulation. Applied Physics Let- 
ters, 104, Article ID: 252409.http://dx.doi.org/10.1063/1.4885146

[9] Murase, K., Oonoki, J., Takata, H., Song, R., Angraini, A.,Ausanai, P. and Matsushita, T. (2011) Simulation and Experimental Studies on Magnetic Hyperthermia with Use of Superparamagnetic Iron Oxide Nanoparticles. Radiological Physics and Technology, 4, 194-202. http://dx.doi.org/10.1007/s12194-011-0123-4

[10] Biederer, S., Knopp, T., Sattel, T.F., Ludtke-Buzug, K., Gleich, B., Weizenecker, J., Borgert, J. and Buzug, T.M. (2009) Magnetization Response Spectroscopy of Superparamagnetic Nanoparticles for Magnetic Particle Imaging. Journal of Physics D: Applied Physics, 42, Article ID: 205007. http://dx.doi.org/10.1088/0022-3727/42/20/205007

[11] Markov, D.E., Boeve, H., Gleich, B., Borgert, J., Antonelli, A., Sfara, C. and Magnani, M. (2010) Human Erythrocytes as Nanoparticle Carriers for Magnetic Particle Imaging. Physics in Medicine and Biology, 55, 6461-6473. http://dx.doi.org/10.1088/0031-9155/55/21/008

[12] Murase, K., Banura, N., Mimura, A. and Nishimoto, K. (2015) Simple and Practical Method for Correcting the Inhomogeneous Sensitivity of a Receiving Coil in Magnetic Particle Imaging. Japanese Journal of Applied Physics, 54, Article ID: 038001.http://dx.doi.org/10.7567/JJAP.54.038001

[13] Nelder, J.A. and Mead, R. (1965) A Simplex Method for Function Minimization. The Computer Journal, 7, $308-313$. http://dx.doi.org/10.1093/comjnl/7.4.308

[14] Semmler-Behnke, M., Takenaka, S., Fertsch, S., Wenk, A., Seitz, J., Mayer, P., Oberdöster, G. and Kreyling, W.G. (2007) Efficient Elimination of Inhaled Nanoparticles from the Alveolar Region: Evidence for Interstitial Uptake and Subsequent Reentrainment onto Airways Epithelium. Environmental Health Perspectives, 115, 728-733. http://dx.doi.org/10.1289/ehp.9685

[15] Semmler, M., Seitz, J., Erbe, F., Mayer, P., Heyder, J., Oberdörster, G. and Kreyling, W.G. (2004) Long-Term Clearance Kinetics of Inhaled Ultrafine Insoluble Iridium Particles from the Rat Lung, Including Transient Translocation into Secondary Organs. Inhalation Toxicology, 16, 453-459. http://dx.doi.org/10.1080/08958370490439650

[16] Fleming, J.S., Quint, M., Bolt, L., Martonen, T.B. and Conway, J.H. (2006) Comparison of SPECT Aerosol Deposition Data with Twenty-Four-Hour Clearance Measurements. Journal of Aerosol Medicine, 19, 261-267. http://dx.doi.org/10.1089/jam.2006.19.261

[17] Robson, M.D., Gatehouse, P.D., Bydder, M. and Bydder, G.M. (2003) Magnetic Resonance: An Introduction to Ultrashort TE (UTE) Imaging. Journal of Computer Assisted Tomography, 27, 825-846. http://dx.doi.org/10.1097/00004728-200311000-00001

[18] Idiyatullin, D., Corum, C., Park, J.Y. and Garwood, M. (2006) Fast and Quiet MRI Using a Swept Radiofrequency. Journal of Magnetic Resonance, 181, 342-349. http://dx.doi.org/10.1016/i.jmr.2006.05.014

[19] Zhang, J.J., Chamberlain, R., Etheridge, M., Idiyatullin, D., Corum, C., Bischof, J. and Garwood, M. (2014) Quantifying Iron-Oxide Nanoparticles at High Concentration Based on Longitudinal Relaxation Using a Three-dimensional SWIFT Look-Locker Sequence. Magnetic Resonance in Medicine, 71, 1982-1988. http://dx.doi.org/10.1002/mrm.25181

[20] Hoopes, P.J., Petryk, A.A., Tate, J.A., Savellano, M.S., Strawbridge, R.R., Giustini, A.J., Stan, R.V., Gimi, B. and Garwood, M. (2013) Imaging and Modification of the Tumor Vascular Barrier for Improvement in Magnetic Nanoparticle Uptake and Hyperthermia Treatment Efficacy. Proceedings SPIE 8584, Energy-Based Treatment of Tissue and Assessment VII, 858403, San Francisco, 26 February 2013, 1-14. http://dx.doi.org/10.1117/12.2008689

[21] Möller, W., Häußinger, K., Winkler-Heil, R., Stahlhofen, W., Meyer, T., Hofmann, W. and Heyder, J. (2004) Mucociliary and Long-Term Particle Clearance in the Airways of Healthy Nonsmoker Subjects. Journal of Applied Physiology, 97, 2200-2206. http://dx.doi.org/10.1152/japplphysiol.00970.2003

[22] Plank, C. (2008) Nanomagnetosols: Magnetism Opens up New Perspectives for Targeted Aerosol Delivery to the Lung. Trends in Biotechnology, 26, 59-63. http://dx.doi.org/10.1016/j.tibtech.2007.11.001 